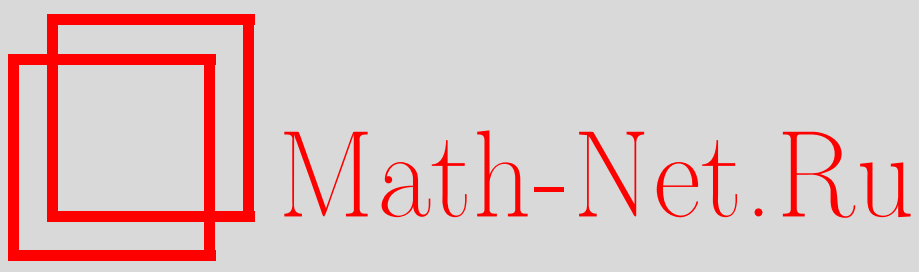

V. A. Molchanov, Elementary definability of the class of universal planar automata in the class of semigroups, Sibirsk. Mat. Zh., 2019, Volume 60, Number 6, 1397-1410

DOI: https://doi.org/10.33048/smzh.2019.60.616

Use of the all-Russian mathematical portal Math-Net.Ru implies that you have read and agreed to these terms of use http://www . mathnet.ru/eng/agreement

Download details:

IP: 54.197 .217 .227

April 26, 2023, 16:36:35

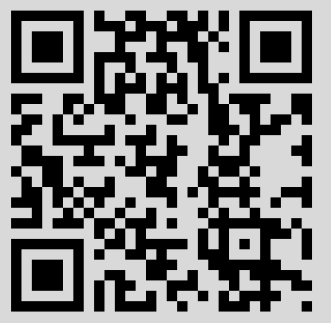


Сибирский математический журнал

Ноябрь-декабрь, 2019. Том 60, № 6

УДК 512.534.5+519.713.2

\title{
ЭЛЕМЕНТАРНАЯ ОПРЕДЕЛИМОСТЬ КЛАССА УНИВЕРСАЛЬНЫХ ПЛАНАРНЫХ АВТОМАТОВ В КЛАССЕ ПОЛУГРУПП
}

\section{В. А. Молчанов}

\begin{abstract}
Аннотация. Универсальные планарные автоматы являются универсальными притягивающими объектами в категории полугрупповых автоматов, у которых множества состояний и множества выходных сигналов наделены алгебраическими структурами плоскостей, инвариантными относительно действий функций перехода и выхода. Доказана элементарная определимость класса универсальных планарных автоматов в классе полугрупп и исследована проблема элементарной классификации универсальных планарных автоматов с помощью теорий первого порядка полугрупп входных сигналов таких автоматов.
\end{abstract}

DOI 10.33048/smzh.2019.60.616

Ключевые слова: элементарная определимость, автомат, плоскость, полугруппа, элементарная классификация.

\section{1. Введение}

Одним из главных разделов современной алгебры является обобщенная теория Галуа, начало которой было положено в исследованиях Э. Галуа и которая посвящается изучению математических объектов путем исследования некоторых производных алгебраических систем, специальным образом связанных с исходными объектами. В качестве исходных математических объектов рассматривались разнообразные алгебраические системы (от графов и гиперграфов до колец и векторных пространств), топологические пространства, формальные языки и многие другие, а в качестве производных алгебраических систем рассматривались, соответственно группы автоморфизмов, полугруппы эндоморфизмов и решетки подсистем алгебраических систем, группы гомеоморфизмов и полугруппы непрерывных преобразований топологических пространств, синтаксические моноиды формальных языков и многие другие. Проводимые в этом направлении исследования обычно следуют уже сложившемуся традиционному кругу вопросов обобщенной теории Галуа: принципиальное значение имеет задача о том, насколько хорошо производная алгебраическая система определяет исходный математический объект; затем исследуется, какими абстрактными и элементарными свойствами характеризуется такая производная алгебраическая система; наконец, с помощью полученных результатов изучаются взаимосвязи свойств исходного объекта и его производной алгебраической системы. Такие вопросы для групп автоморфизмов алгебраических систем, полугрупп эндоморфизмов графов, колец эндоморфизмов модулей и других производных алгебраических систем весьма успешно решались Б. И. Плоткиным [1], А. Г. Пинусом

(с) 2019 Молчанов В. А. 
[2-4], Л. М. Глускиным [5,6], Ю. М. Важениным [7,8], А. В. Михалевым [9] и многими другими алгебраистами.

В обзорной статье Ю. М. Важенина и А. Г. Пинуса [10] отмечается, что одними из важнейших проблем обобщенной теории Галуа являются проблема элементарной классификации исходных объектов с помощью теорий первого порядка производных алгебраических систем и проблема разрешимости теорий первого порядка производных алгебраических систем. В этом направлении важные результаты получены А. Г. Пинусом [2-4] для разнообразных производных структур свободных алгебр, унаров и групп, Ю. М. Важениным $[7,8]$ для полугрупп преобразований графов, Е. И. Буниной и А. В. Михалевым [11] для колец эндоморфизмов групп, Ф. А. Мурзиным для колец непрерывных функций [12]. Как известно [13], эффективным инструментом решения такого рода проблем является метод относительно элементарной определимости одного класса моделей $\mathbf{K}$ в другом классе моделей $\mathbf{K}_{\mathbf{1}}$, суть которого заключается в построении изоморфной копии исходной модели $A \in \mathbf{K}$ в ее производной модели $S(A) \in \mathbf{K}_{\mathbf{1}}$ с помощью средств узкого исчисления предикатов (УИП) сигнатуры класса $\mathbf{K}_{\mathbf{1}}$ и некоторых фиксированных элементов модели $S(A)$. Если при этом изоморфная копия модели $A$ в модели $S(A)$ строится с помощью средств узкого исчисления предикатов (УИП) без использования фиксированных элементов модели $S(A)$, то относительно элементарную определимость принято в теории моделей называть элементарной определимостью.

K обобщенной теории Галуа относятся также исследования автоматов в категориях [14], т. е. автоматов, у которых системы состояний и выходных сигналов являются объектами некоторой категории $\mathbf{K}$, а функции переходов и выходные функции - морфизмами категории К. Например, Л. М. Глускин, Ю. М. Важенин и другие авторы (см., обзор в [15]) исследовали полугруппы эндоморфизмов графов, которые могут рассматриваться как графические полуавтоматы (т. е. автоматы без выходных сигналов, для которых системы состояний являются объектами категории графов $\mathbf{G r}$ ). Кроме того, известная проблема Л. М. Глускина и Л. А. Скорнякова о полугруппах эндоморфизмов проективных плоскостей может быть рассмотрена как проблема определимости планарных полуавтоматов их полугруппами входных сигналов (см. проблему 1.20 в [16]). В [17] было исследовано обобщение этой проблемы на автоматы с выходными сигналами, для которых системы состояний и выходных сигналов являются объектами категории плоскостей Pl. Как следует из [14], в категории планарных автоматов для любых плоскостей П, $\Pi^{\prime} \in \mathbf{P l}$ существует универсальный притягивающий объект $\operatorname{Atm}\left(\Pi, \Pi^{\prime}\right)$, который называется универсальным планарным автоматом над плоскостями П, $\Pi^{\prime}$. Полугруппа входных сигналов такого автомата $S=\operatorname{End}\left(\Pi_{1}\right) \times \operatorname{Hom}\left(\Pi_{1}, \Pi_{2}\right)$ рассматривается в качестве производной алгебраической системы автомата $\operatorname{Atm}\left(\Pi, \Pi^{\prime}\right)$. Следовательно, алгебраическая теория планарных автоматов тесным образом связана как с обобщенной теорией Галуа, так и с общеизвестной задачей определяемости математических объектов их автоморфизмами и эндоморфизмами, которая сформулирована в числе прочих актуальных математических проблем в книге С. Улама [18]. Главный результат работы [17] показывает, что универсальные планарные автоматы определяются с точностью до изоморфизма своими полугруппами входных сигналов. Для исследования проблемы элементарной определимости таких автоматов своими полугруппами входных сигналов в настоящей работе доказывается элементарная определимость класса универсальных планарных автома- 
тов в классе полугрупп. Полученный результат дает возможность проанализировать взаимосвязь важных свойств элементарных теорий классов планарных автоматов и элементарных теорий классов полугрупп таких, как проблема элементарной эквивалентности алгебраических систем, проблема алгоритмической разрешимости элементарных теорий классов алгебраических систем и др.

\section{2. Основные понятия}

Вначале напомним некоторые понятия из математической логики, теории плоскостей и теории автоматов, которые будут использоваться ниже. Читатели, интересующиеся дальнейшими деталями, могут обратиться, например, к работам Ю. Л. Ершова и Е. А. Палютина [19], Р. Хартсхорна [20] и Б. И. Плоткина [14].

Под отображением множества $X$ в множество $Y$, обозначаемым $\varphi: X \rightarrow Y$, понимается однозначное бинарное отношение $\varphi \subset X \times Y$ с областью определения $\operatorname{dom} \varphi=X$. Для элемента $x \in X$ образ $x$ относительно отображения $\varphi$ обозначается через $\varphi(x)$. Для подмножества $A \subset X$ положим $\varphi(A)=\{\varphi(x) \mid x \in A\}$. Для элемента $a \in Y$ символом $\xi_{a}$ обозначается постоянное отображение $\xi_{a}: X \rightarrow\{a\}$. Отображение $\varphi: X \rightarrow X$ называется преобразованием множества $X$. Символом $\Delta_{X}$ обозначается тождественное преобразование множества $X$.

Для отображений $\varphi: X \rightarrow Y, \psi: Y \rightarrow Z$ композиция $\varphi \psi: X \rightarrow Z$ определяется по формуле $\varphi \psi(x)=\psi(\varphi(x))$; для отображения $\varphi: X \rightarrow Y$ декартова степень $\varphi^{3}: X^{3} \rightarrow Y^{3}-$ по формуле $\varphi^{3}\left(x_{1}, x_{2}, x_{3}\right)=\left(\varphi\left(x_{1}\right), \varphi\left(x_{2}\right), \varphi\left(x_{3}\right)\right)$.

Пусть $X, Y$ - непустые множества. Обозначим через $T(X)$ множество всех преобразований $X, F(X, Y)$ - множество всех отображений $X$ в $Y$ и $S(X, Y)-$ декартово произведение $T(X) \times F(X, Y)$ множеств $T(X), F(X, Y)$. Элементами множества $S(X, Y)$ являются упорядоченные пары $\left(f_{1}, f_{2}\right)$ отображений $f_{1}$ : $X \rightarrow X, f_{2}: X \rightarrow Y$, которые могут рассматриваться как вектор-функции $f: X \rightarrow X \times Y$, определяющиеся по формуле $f(x)=\left(f_{1}(x), f_{2}(x)\right)$ для $x \in X$. Отображения $f_{1}, f_{2}$ называются соответственно первой и второй компонентами вектор-функиии $f$. Таким образом, вектор-функции могут отождествляться с упорядоченными парами их компонент.

Следуя [14], для вектор-функций $f, g \in S(X, Y)$ определяется произведение $f \cdot g \in S(X, Y)$ по формуле $f \cdot g=\left(f_{1}, f_{2}\right) \cdot\left(g_{1}, g_{2}\right)=\left(f_{1} g_{1}, f_{1} g_{2}\right)$. Так как эта операция ассоциативна [14], множество $S(X, Y)$ с операцией умножения векторфункций образует полугруппу.

Следуя [20], под плоскостью будем понимать алгебраическую систему вида $\Pi=(X, L)$, где $X$ - непустое множество точек и $L-$ семейство его подмножеств, именуемых прямыми, удовлетворяющее следующим аксиомам: $\left(A_{1}\right)$ любые две точки плоскости П содержатся в одной и только одной прямой; $\left(A_{2}\right)$ любая прямая плоскости П содержит по крайней мере три точки; $\left(A_{3}\right)$ в плоскости П существуют три точки, не принадлежащие одной прямой. Точки плоскости называются коллинеарными, если они лежат на одной прямой.

В частности, плоскость П является проективной, если любые две ее прямые имеют общую точку, и аффинной, если для любой прямой $l \in L$ и любой точки $x \in X \backslash l$ существует такая единственная прямая $l_{1}$, что $x \in l_{1}$ и $l \cap l_{1}=\varnothing$.

Пусть $\Pi=(X, L), \Pi^{\prime}=\left(X^{\prime}, L^{\prime}\right)$ - некоторые плоскости. Отображение $\varphi: X \rightarrow X^{\prime}$ называется гомоморфизмом П в $\Pi^{\prime}$ и обозначается через $\varphi: \Pi \rightarrow \Pi^{\prime}$, если оно отображает коллинеарные точки плоскости П в коллинеарные точки 
плоскости П', т. е. выполняется следующее условие:

$$
(\forall l \in L)\left(\exists l^{\prime} \in L^{\prime}\right) \varphi(l) \subset l^{\prime} .
$$

Множество всех гомоморфизмов П в $\Pi^{\prime}$ обозначим через $\operatorname{Hom}\left(\Pi, \Pi^{\prime}\right)$. Очевидно, что для любой прямой $l^{\prime}$ плоскости $\Pi^{\prime}$ любое отображение $\varphi: X \rightarrow l^{\prime}$ является гомоморфизмом П в $\Pi^{\prime}$.

Гомоморфизм плоскости П в себя называется эндоморфизмом П. Множество всех эндоморфизмов плоскости П с операцией композиции образует полугруппу End П.

Для плоскостей $\Pi=(X, L), \Pi^{\prime}=\left(X^{\prime}, L^{\prime}\right)$ множество End $\Pi \times \operatorname{Hom}\left(\Pi, \Pi^{\prime}\right)$ с операцией умножения вектор-функций образует полугруппу, которую обозначим через $S\left(\Pi, \Pi^{\prime}\right)$.

Для описания свойств плоскости $\Pi=(X, L)$ на языке УИП будем рассматривать П как двухсортную алгебраическую систему $\Pi=(X, L, \rho)$ с двумя базисными множествами $X, L$ и бинарным отношением $\rho \subset X \times L$, которое для элементов $x \in X, l \in L$ определяется по формуле $(x, l) \in \rho \Longleftrightarrow x \in l$ и удовлетворяет аксиомам плоскости:

$$
\begin{gathered}
\left(\forall x^{(1)}, y^{(1)}\right)\left(x^{(1)} \neq y^{(1)} \Longrightarrow\left(\exists ! x^{(2)}\right)\left(\left(x^{(1)}, x^{(2)}\right) \in \rho \wedge\left(y^{(1)}, x^{(2)}\right) \in \rho\right)\right), \\
\left(\forall x^{(2)}\right)\left(\exists x_{1}^{(1)}, x_{2}^{(1)}, x_{3}^{(1)}\right)\left(\bigwedge_{1 \leq i \neq j \leq 3} x_{i}^{(1)} \neq x_{j}^{(1)} \wedge \bigwedge_{1 \leq i \leq 3}\left(x_{i}^{(1)}, x^{(2)}\right) \in \rho\right), \\
\left(\exists x_{1}^{(1)}, x_{2}^{(1)}, x_{3}^{(1)}\right)\left(\forall x^{(2)}\right) \neg\left(\bigwedge_{1 \leq i \leq 3}\left(x_{i}^{(1)}, x^{(2)}\right) \in \rho\right),
\end{gathered}
$$

где $x^{(1)}$ и $x^{(2)}$ - переменные первого и второго сорта, обозначающие соответственно точки и прямые плоскости.

В частности, плоскость $\Pi=(X, L, \rho)$ является проективной, если выполняется аксиома

$$
\left(\forall x^{(2)}, y^{(2)}\right)\left(\exists x^{(1)}\right)\left(\left(x^{(1)}, x^{(2)}\right) \in \rho \wedge\left(x^{(1)}, y^{(2)}\right) \in \rho\right),
$$

и аффинной, если выполняется аксиома

$$
\begin{aligned}
& \left(\forall x^{(2)}\right)\left(\forall x^{(1)}\right)\left(\left(x^{(1)}, x^{(2)}\right) \notin \rho\right. \\
& \left.\quad \Rightarrow\left(\exists ! y^{(2)}\right)\left(\left(x^{(1)}, y^{(2)}\right) \in \rho \wedge\left(\forall y^{(1)}\right)\left(\left(y^{(1)}, x^{(2)}\right) \notin \rho \vee\left(y^{(1)}, y^{(2)}\right) \notin \rho\right)\right)\right) .
\end{aligned}
$$

Изоморфизмом таких алгебраических систем $\Pi=(X, L, \rho), \Pi^{\prime}=\left(X^{\prime}, L^{\prime}, \rho^{\prime}\right)$ является упорядоченная пара $\pi=(\varphi, \psi)$ биекций $\varphi: X \rightarrow X^{\prime}, \psi: L \rightarrow L^{\prime}$, сохраняющая бинарные отношения этих систем, т. е. для любых $x \in X, l \in L$ выполняется условие

$$
(x, l) \in \rho \Longleftrightarrow(\varphi(x), \psi(l)) \in \rho^{\prime} .
$$

Следуя [14], под автоматом будем понимать алгебраическую систему $\mathbf{A}=$ $(X, S, Y, \delta, \lambda)$, состоящую из множества состояний $X$, полугруппы входных сигналов $S=\operatorname{Inp}(\mathbf{A})$, множества выходных сигналов $Y$, функции переходов $\delta$ : $X \times S \rightarrow X$ и выходной функции $\lambda: X \times S \rightarrow Y$ таких, что для любых $x \in X$ и $s, t \in S$ выполняются условия $\delta(x, s t)=\delta(\delta(x, s), t), \lambda(x, s t)=\lambda(\delta(x, s), t)$.

Для каждого $s \in S$ определим отображения $\delta_{s}: X \rightarrow X, \lambda_{s}: X \rightarrow Y$ и вектор-функцию $f_{s}=\left(\delta_{s}, \lambda_{s}\right)$ по формулам $\delta_{s}(x)=\delta(x, s), \lambda_{s}(x)=\lambda(x, s)$, где 
$x \in X$. По определению автомата последовательное действие входных сигналов $s, t \in S$ удовлетворяет следующим условиям:

$$
f_{t}\left(f_{s}(x)\right)=(\delta(\delta(x, s), t), \lambda(\delta(x, s), t))=(\delta(x, s t), \lambda(x, s t))=f_{s t}(x) .
$$

Это означает, что соответствие $s \mapsto f_{s}(s \in S)$ является гомоморфизмом полугруппы $S$ в полугруппу вектор-функций $S(X, Y)$. В частности, это соответствие является мономорфизмом полугруппы $S$ в полугруппу $S(X, Y)$, если $\mathbf{A}=(X, S, Y, \delta, \lambda)$ является автоматом без равнодействующих сигналов, т. е. для любых $s, t \in S, s \neq t$ выполняется условие $f_{s} \neq f_{t}$. В этом случае действие входного сигнала $s \in S$ полностью определяется действием пары отображений $\delta_{s}, \lambda_{s}$ и можно отождествить входной сигнал $s$ с вектор-функцией $f_{s}=\left(\delta_{s}, \lambda_{s}\right)$.

Входной сигнал $a \in S$ автомата $\mathbf{A}=(X, S, Y, \delta, \lambda)$ называется автономным, если его действие не зависит от состояний этого автомата, т. е. найдутся такие состояние автомата, обозначаемое через $a_{1}$, и выходной сигнал автомата, обозначаемый через $a_{2}$, что $\delta(x, a)=a_{1}, \lambda(x, a)=a_{2}$ для всех состояний автомата $x \in X$. Для автомата $\mathbf{A}$ без равнодействующих входных сигналов автономный входной сигнал $a=\left(\delta_{a}, \lambda_{a}\right)=\left(\xi_{a_{1}}, \xi_{a_{2}}\right)$ является упорядоченной парой постоянных отображений $\xi_{a_{1}}: X \rightarrow\left\{a_{1}\right\}, \xi_{a_{2}}: X \rightarrow\left\{a_{2}\right\}$.

По определению [14] планарные автоматы являются автоматами в категории плоскостей $\mathbf{P l}$, т. е. автоматами $\mathbf{A}=\left(X_{1}, S, X_{2}, \delta, \lambda\right)$ с множеством состояний $X_{1}$ и множеством выходных сигналов $X_{2}$, наделенными структурами плоскостей $\Pi_{1}=\left(X_{1}, L_{1}\right)$ и $\Pi_{2}=\left(X_{2}, L_{2}\right)$, полугруппой входных сигналов $S=\operatorname{Inp}(\mathbf{A})$, функцией переходов $\delta: X_{1} \times S \rightarrow X_{1}$ и выходной функцией $\lambda: X_{1} \times S \rightarrow X_{2}$, для которых при каждом фиксированном $s \in S$ преобразование $\delta_{s}: X_{1} \rightarrow X_{1}$ является эндоморфизмом плоскости П 1 и отображение $\lambda_{s}: X_{1} \rightarrow X_{2}$ является гомоморфизмом плоскости $\Pi_{1}$ в плоскость $\Pi_{2}$. Такие автоматы обозначаются символом $\mathbf{A}=\left(\Pi_{1}, S, \Pi_{2}, \delta, \lambda\right)$.

Так как с алгебраической точки зрения планарные автоматы представляют собой многосортные алгебраические системы, гомоморфизмом планарного автомата $\mathbf{A}=\left(\Pi_{X}, S, \Pi_{Y}, \delta, \lambda\right)$ в планарный автомат $\mathbf{A}^{\prime}=\left(\Pi_{X^{\prime}}, S^{\prime}, \Pi_{Y^{\prime}}, \delta^{\prime}, \lambda^{\prime}\right)$ называется упорядоченная тройка $\gamma=(f, \pi, g)$ отображений $f: X \rightarrow X^{\prime}$, $\pi: S \rightarrow S^{\prime}$ и $g: Y \rightarrow Y^{\prime}$, сохраняющих алгебраическую структуру таких автоматов, т. е. $f$ является гомоморфизмом плоскости $\Pi_{X}$ в плоскость $\Pi_{X^{\prime}}, \pi-$ гомоморфизмом полугруппы $S$ в полугруппу $S^{\prime}, g$ - гомоморфизмом плоскости $\Pi_{Y}$ в плоскость П $Y_{Y^{\prime}}$, и для любых значений $x \in X, s \in S$ выполняются условия

$$
f(\delta(x, s))=\delta^{\prime}(f(x), \pi(s)), \quad g(\lambda(x, s))=\lambda^{\prime}(f(x), \pi(s)) .
$$

Важный пример планарного автомата дает алгебраическая система

$$
\operatorname{Atm}\left(\Pi_{X}, \Pi_{Y}\right)=\left(\Pi_{X}, S\left(\Pi_{X}, \Pi_{Y}\right), \Pi_{Y}, \delta^{\circ}, \lambda^{\circ}\right),
$$

где $\Pi_{X}=\left(X, L_{X}\right), \Pi_{Y}=\left(Y, L_{Y}\right)$ - некоторые плоскости и для любых $x \in X$, $(\varphi, \psi) \in S\left(\Pi_{X}, \Pi_{Y}\right)$ определены значения $\delta^{\circ}(x,(\varphi, \psi))=\varphi(x), \lambda^{\circ}(x,(\varphi, \psi))=$ $\psi(x)$.

Легко проверить, что $\operatorname{Atm}\left(\Pi_{X}, \Pi_{Y}\right)$ удовлетворяет следующему универсальному свойству: для любого планарного автомата $\mathbf{A}=\left(\Pi_{X}, S, \Pi_{Y}, \delta, \lambda\right)$ существует гомоморфизм $\pi: S \rightarrow S\left(\Pi_{X}, \Pi_{Y}\right)$ такой, что упорядоченная тройка $\gamma=\left(\Delta_{X}, \pi, \Delta_{Y}\right)$ является гомоморфизмом $\mathbf{A}$ в $\operatorname{Atm}\left(\Pi_{X}, \Pi_{Y}\right)$. По этой причине автомат $\operatorname{Atm}\left(\Pi_{X}, \Pi_{Y}\right)$ называется [14] универсальным планарным автоматом над плоскостлми $\Pi_{X}, \Pi_{Y}$. 
Для описания свойств планарных автоматов $\mathbf{A}=\left(\Pi_{1}, S, \Pi_{2}, \delta, \lambda\right)$ на языке УИП будем рассматривать $\mathbf{A}$ в виде пятисортной алгебраической системы $\mathbf{A}=\left(\left(X_{1}, L_{1}, \epsilon_{1}\right),(S, \cdot),\left(X_{2}, L_{2}, \in_{2}\right), \delta, \lambda\right)$ с пятью базисными множествами $X_{1}, L_{1}, S, X_{2}, L_{2}$ и сигнатурой $\Omega=\left\{\epsilon_{1}, \cdot, \in_{2}, \delta, \lambda\right\}$. Напомним, что здесь $X_{1}$ и $L_{1}$ (соответственно $X_{2}$ и $L_{2}$ ) - множества точек и прямых плоскости состояний $\Pi_{1}$ (соответственно плоскости выходных сигналов $\left.\Pi_{2}\right), S$ - множество входных сигналов автомата $\mathbf{A}, \epsilon_{1}$ (соответственно $\epsilon_{2}$ ) - символ бинарного отношения принадлежности точек плоскости состояний П ходных сигналов $\Pi_{2}$ ) ее прямым, - - символ бинарной операции полугруппы $S$, $\delta$ и $\lambda$ - символы бинарных операций функции переходов и выходной функции автомата $\mathbf{A}$.

Элементарная теория планарных автоматов определяется в стиле аксиоматики Гильберта геометрии плоскости с помощью языка УИП сигнатуры $\Omega$ с пятисортными переменными $\mathbf{L}_{\mathbf{A}}$. Алфавит такого языка состоит: (1) из счетного множества индивидуальных переменных первого сорта $x^{(1)}, y^{(1)}, \ldots$ (соответственно второго сорта $x^{(2)}, y^{(2)}, \ldots$ ) для обозначения точек (соответственно прямых) плоскости состояний автомата; (2) из счетного множества индивидуальных переменных третьего сорта $x^{(3)}, y^{(3)}, \ldots$ для обозначения входных сигналов автомата; (3) из счетного множества индивидуальных переменных четвертого сорта $x^{(4)}, y^{(4)}, \ldots$ (соответственно пятого сорта $\left.x^{(5)}, y^{(5)}, \ldots\right)$ для обозначения точек (соответственно прямых) плоскости выходных сигналов автомата; (4) из двуместного предикатного символа $\epsilon_{1}$ (соответственно $\epsilon_{2}$ ) для обозначения отношения принадлежности точек плоскости состояний (соответственно плоскости выходных сигналов) автомата ее прямым; (5) из двуместного функционального символа · для обозначения операции умножения полугруппы входных сигналов автоматов; (6) из двуместных функциональных символов $\delta$ и $\lambda$ для обозначения функции переходов и выходной функции автомата; (7) из конечного множества логических и технических символов таких, как $\neg, \wedge, \vee, \Longrightarrow, \Longleftrightarrow, \forall, \exists,=,($,$) .$

Для языка $\mathbf{L}_{\mathbf{A}}$ термы трех сортов получаются обычным комбинированием символа - с двумя термами третьего сорта и символов $\delta, \lambda$ с термами первого и третьего сортов, т. е. это выражения вида $x^{(1)}, x^{(2)}, x^{(3)}, x^{(4)}, t^{(3)} \cdot t_{1}^{(3)}$, $\delta\left(t^{(1)}, t^{(3)}\right), \lambda\left(t^{(1)}, t^{(3)}\right)$, где $x^{(1)}$ и $t^{(1)}$ - переменная и терм первого сорта, $x^{(2)}-$ переменная второго сорта, $x^{(3)}$ и $t^{(3)}, t_{1}^{(3)}$ - переменная и термы третьего сорта. При этом получаются термы $\delta\left(t^{(1)}, t^{(3)}\right), t^{(3)} \cdot t_{1}^{(3)}$ и $\lambda\left(t^{(1)}, t^{(3)}\right)$ первого, третьего и четвертого сортов соответственно. Термами второго и пятого сортов являются индивидуальные переменные второго и пятого сортов, обозначающие прямые, соответственно плоскости состояний и плоскости выходных сигналов автоматов.

Атомарные формулы языка $\mathbf{L}_{\mathbf{A}}$ получаются обычным комбинированием символа $=$ с двумя термами одного сорта, символа $\epsilon_{1}-$ с термом первого сорта и термом второго сорта и символа $\epsilon_{2}-$ с термом четвертого сорта и термом пятого сорта, т. е. это выражения вида $t=t^{\prime}, t^{(1)} \in_{1} t^{(2)}$ и $t^{(4)} \in_{2} t^{(5)}$, где $t, t^{\prime}-$ термы одного и того же сорта, $t^{(i)}$ - термы $i$-го сорта (где $\left.i=1,2,4,5\right)$. Формулы языка $\mathbf{L}_{\mathbf{A}}$ определяются по индукции обычным образом (см., например, [19]).

\section{3. Вспомогательные результаты}

Согласно [21] для универсального планарного автомата $\mathbf{A}=\operatorname{Atm}\left(\Pi_{1}, \Pi_{2}\right)$ над плоскостями $\Pi_{1}=\left(X_{1}, L_{1}\right)$ и $\Pi_{2}=\left(X_{2}, L_{2}\right)$ естественно определяются сле- 
дующие канонические отношения на полугруппе входных сигналов $S=\operatorname{Inp}(\mathbf{A})$ :

1) множество $C$ всех автономных входных сигналов $a$ автомата $\mathbf{A}$, под действием которых все состояния автомата переводятся в одно и то же состояние $a_{1} \in X_{1}$ и отображаются в один и тот же выходной сигнал $a_{2} \in X_{2}$;

2 ) бинарное отношение $\varepsilon_{1}$ на множестве $C$, которое состоит из таких упорядоченных пар $(a, b)$ автономных входных сигналов $a, b \in C$, действия которых одинаково преобразуют состояния автомата $\mathbf{A}$, т. е. по определению $(a, b) \in \varepsilon_{1} \Longleftrightarrow a_{1}=b_{1}$;

3) бинарное отношение $\varepsilon_{2}$ на множестве $C$, которое состоит из таких упорядоченных пар $(a, b)$ автономных входных сигналов $a, b \in C$, при действии которых состояния автомата $\mathbf{A}$ отображаются в одинаковые выходные сигналы, т. е. по определению $(a, b) \in \varepsilon_{2} \Longleftrightarrow a_{2}=b_{2}$;

4) бинарное отношение $\eta$ на множестве $C^{2}$, которое состоит из таких упорядоченных пар $(\alpha, \beta)$ элементов $\alpha=(a, b)$ и $\beta=(c, d)$ с автономными входными сигналами $a, b, c, d \in C$, при действии которых для каждого $i=1,2$ состояния автомата $\mathbf{A}$ отображаются в коллинеарные точки $a_{i}, b_{i}, c_{i}, d_{i}$ соответствующей плоскости $\Pi_{i}$, т. е. по определению

$$
(\alpha, \beta) \in \eta \Longleftrightarrow \text { точки } a_{i}, b_{i}, c_{i}, d_{i} \text { колинеарны в плоскости } \Pi_{i}(i=1,2) .
$$

В [21] доказано, что канонические отношения универсального планарного автомата $\mathbf{A}=\operatorname{Atm}\left(\Pi_{1}, \Pi_{2}\right)$ удовлетворяют следующим условиям:

1) для любого состояния (соответственно выходного сигнала) $x$ автомата A найдется такой автономный входной сигнал этого автомата, обозначаемый через $\hat{x}$, при действии которого все состояния автомата переходят в состояние $x$ (соответственно отображаются в выходной сигнал $x$ ), т. е. выполняется равенство $\hat{x}_{1}=x$ (соответственно $\left.\hat{x}_{2}=x\right)$;

2) для каждого $i=1,2$ отношение $\varepsilon_{i}$ является эквивалентностью на множестве $C$ и отображение $\varphi_{i}: X_{i} \rightarrow C / \varepsilon_{i}$, определяемое для элементов $x \in X_{i}$ по формуле $\varphi_{i}(x)=\varepsilon_{i}(\hat{x})$, является биекцией $X_{i}$ на фактор-множество $C / \varepsilon_{i}$;

3 ) для каждого $i=1,2$ ограничение $\eta_{i}$ отношения $\eta$ на множестве $D_{i}=$ $C^{2} \backslash \varepsilon_{i}$ является эквивалентностью такой, что отображение $\psi_{i}: L_{i} \rightarrow D_{i} / \eta_{i}$, определяемое для элементов $l \in L_{i}$ по формуле $\psi_{i}(l)=\eta_{i}(\hat{x}, \hat{y})$ для произвольных различных точек $x, y \in l$, является биекцией $L_{i}$ на фактор-множество $D_{i} / \eta_{i}$.

Пусть $\mathbf{A}=\operatorname{Atm}\left(\Pi_{1}, \Pi_{2}\right)$ - универсальный планарный автомат над некоторыми плоскостями $\Pi_{1}=\left(X_{1}, L_{1}\right)$ и $\Pi_{2}=\left(X_{2}, L_{2}\right)$. С помощью канонических отношений этого автомата введем следующие понятия:

1) для каждого $i=1,2$ определим двухсортную алгебраическую систему $\bar{\Pi}_{i}=\left(\bar{X}_{i}, \bar{L}_{i}, \bar{\rho}_{i}\right)$ с базисными множествами $\bar{X}_{i}=C / \varepsilon_{i}, \bar{L}_{i}=D_{i} / \eta_{i}$ и бинарным отношением $\bar{\rho}_{i} \subset \bar{X}_{i} \times \bar{L}_{i}$, которое для элементов $a, b, c \in C, b \not \equiv c\left(\varepsilon_{i}\right)$ задается по формуле

$$
\left(\varepsilon_{i}(a), \eta_{i}(b, c)\right) \in \bar{\rho}_{i} \quad \Longleftrightarrow \quad \text { точки } a_{i}, b_{i}, c_{i} \text { коллинеарны в плоскости } \Pi_{i} ;
$$

2) определим два отображения $\bar{\delta}: \bar{X}_{1} \times S \rightarrow \bar{X}_{1}, \bar{\lambda}: \bar{X}_{1} \times S \rightarrow \bar{X}_{2}$, которые для элементов $a \in C, s \in S$ задаются по формулам

$$
\bar{\delta}\left(\varepsilon_{1}(a), s\right)=\varepsilon_{1}(a \cdot s), \quad \bar{\lambda}\left(\varepsilon_{1}(a), s\right)=\varepsilon_{2}(a \cdot s) .
$$

В [21] доказан следующий результат. 
Теорема 1. Пусть $\mathbf{A}=\operatorname{Atm}\left(\Pi_{1}, \Pi_{2}\right)$ - универсальный планарный автомат над некоторыми плоскостями $\Pi_{1}=\left(X_{1}, L_{1}\right), \Pi_{2}=\left(X_{2}, L_{2}\right)$. Тогда справедливы следующие утверждения:

1) для каждого $i=1,2$ плоскость $\Pi_{i}$ изоморфна алгебраической системе $\bar{\Pi}_{i}=\left(\bar{X}_{i}, \bar{L}_{i}, \bar{\rho}_{i}\right)$;

2) автомат $\mathbf{A}=\operatorname{Atm}\left(\Pi_{1}, \Pi_{2}\right)$ изоморфен планарному автомату $\overline{\mathbf{A}}=\left(\bar{\Pi}_{1}, S\right.$, $\left.\bar{\Pi}_{2}, \bar{\delta}, \bar{\lambda}\right)$ с плоскостью состояний $\bar{\Pi}_{1}$, полугруппой входных сигналов $S=\operatorname{Inp}(\mathbf{A})$, плоскостью выходных сигналов $\bar{\Pi}_{2}$, функцией переходов $\bar{\delta}: \bar{X}_{1} \times S \rightarrow \bar{X}_{1}$ и выходной функцией $\bar{\lambda}: \bar{X}_{1} \times S \rightarrow \bar{X}_{2}$.

Вышеприведенные результаты показывают, что главным инструментом изучения универсального планарного автомата $\mathbf{A}=\operatorname{Atm}\left(\Pi_{1}, \Pi_{2}\right)$ с помощью исследования его полугруппы входных сигналов $S=\operatorname{Inp}(\mathbf{A})$ являются следующие канонические отношения на этой полугруппе: унарное отношение $C$, бинарные отношения $\varepsilon_{1}, \varepsilon_{2}$ на множестве $C$ и бинарное отношение $\eta$ на множестве $C^{2}$. Покажем, что все эти канонические отношения определяются формулами элементарной теории полугрупп, т. е. формулами языка УИП сигнатуры полугрупп $\mathbf{L}_{\mathbf{S}}$.

Рассмотрим следующие формулы элементарной теории полугрупп:

$$
\begin{gathered}
R Z(x)=(\forall y)(y x=x), \quad L I(x)=(\forall y)(x y=y), \\
E_{1}(x, y)=R Z(x) \wedge R Z(y) \wedge(\forall z)(L I(z) \Longrightarrow x z=y z), \\
R\left(x_{1}, x_{2}, x_{3}\right)=\bigwedge_{i=1}^{3} R Z\left(x_{i}\right) \wedge\left(\forall y_{1}, y_{2}, y_{3}\right)\left(\bigwedge_{i=1}^{3} R Z\left(y_{i}\right)\right. \\
\left.\wedge \bigwedge_{i, j=1, i \neq j}^{3} \neg E_{1}\left(y_{i}, y_{j}\right) \Longrightarrow(\exists z)\left(\bigwedge_{i=1}^{3} y_{i} z=x_{i}\right)\right), \\
E_{2}(x, y)=R Z(x) \wedge R Z(y) \wedge(\forall z)(L I(z) \Longrightarrow R(x, y, x z)), \\
N\left(x_{1}, x_{2}, x_{3}, x_{4}\right)=\bigwedge_{i=1}^{4} R Z\left(x_{i}\right) \wedge \bigwedge_{i, j, k=1}^{4} R\left(x_{i}, x_{j}, x_{k}\right) .
\end{gathered}
$$

Теорема 2. Для канонических отношений любого универсального планарного автомата $\mathbf{A}=\operatorname{Atm}\left(\Pi_{1}, \Pi_{2}\right)$ справедливы следующие утверждения:

1) унарное каноническое отношение $C$ на полугруппе входных сигналов $S=\operatorname{Inp}(\mathbf{A})$ определяется формулой $R Z(x)$;

2) каноническое отношение $\varepsilon_{1}$ на полугруппе входных сигналов $S=\operatorname{Inp}(\mathbf{A})$ определяется формулой $E_{1}(x, y)$;

3) каноническое отношение $\varepsilon_{2}$ на полугруппе входных сигналов $S=\operatorname{Inp}(\mathbf{A})$ определяется формулой $E_{2}(x, y)$;

4) каноническое отношение $\eta$ на полугруппе входных сигналов $S=\operatorname{Inp}(\mathbf{A})$ определяется формулой $N\left(x_{1}, x_{2}, x_{3}, x_{4}\right)$.

ДоказАтельство. Утверждения 1 и 2 доказаны в [17, лемма 2.1, следствие 2.3]. Утверждение 3 доказано в [22, лемма 5]. Утверждение 4 следует из аксиом плоскости и результатов работы [22, лемма 5]. 


\section{4. Основные результаты}

Изложенные в разд. 3 результаты позволяют доказать, что класс Atm всех универсальных планарных автоматов элементарно определим в классе всех полугрупп Sem. Для этого рассмотрим формулы элементарной теории полугрупп, с помощью которых можно построить изоморфную копию любого автомата $\mathbf{A} \in \mathbf{A t m}$ в его полугруппе входных сигналов $\operatorname{Inp}(\mathbf{A}):$

$$
\begin{gathered}
D_{i}(\bar{x})=R Z\left(x_{1}\right) \wedge R Z\left(x_{2}\right) \wedge \neg E_{i}\left(x_{1}, x_{2}\right), \\
\operatorname{Eq}_{i}(\bar{x}, \bar{y})=D_{i}(\bar{x}) \wedge D_{i}(\bar{y}) \wedge N\left(x_{1}, x_{2}, y_{1}, y_{2}\right), \\
\operatorname{In}_{i}(x, \bar{x})=R Z(x) \wedge D_{i}(\bar{x}) \wedge R\left(x, x_{1}, x_{2}\right),
\end{gathered}
$$

где $i=1,2, \bar{x}=\left(x_{1}, x_{2}\right), \bar{y}=\left(y_{1}, y_{2}\right)$.

Теорема 3. Для произвольного универсального планарного автомата $\mathbf{A}=$ $\operatorname{Atm}\left(\Pi_{1}, \Pi_{2}\right)$ над плоскостями $\Pi_{1}=\left(X_{1}, L_{1}\right), \Pi_{2}=\left(X_{2}, L_{2}\right)$ и его полугруппы входных сигналов $S=\operatorname{Inp}(\mathbf{A})$ выполняются следующие утверждения:

1) множества $\widetilde{R Z}=\{s \in S: S \models R Z(s)\}, \widetilde{D_{i}}=\left\{\bar{s} \in S^{2}: S \models D_{i}(\bar{s})\right\}$ $(i=1,2)$ непусты;

$2)$ для каждого $i=1,2$ формула $E_{i}(x, y)$ определяет отношение эквивалентности $\widetilde{E}_{i}$ на множестве $\widetilde{R Z}$;

3) для каждого $i=1,2$ формула $\mathrm{Eq}_{i}(\bar{x} ; \bar{y})$ определяет отношение эквивалентности $\widetilde{\mathrm{Eq}_{i}}$ на множестве $\widetilde{D_{i}}$;

4) для каждого $i=1,2$ формула $\operatorname{In}_{i}(x, \bar{x})$ задает бинарное отношение $\widetilde{\operatorname{In}_{i}}$ между элементами множества $\widetilde{R Z}$ и множества $\widetilde{D_{i}}$, которое согласовано с эквивалентностями $\widetilde{E_{i}}, \widetilde{\mathrm{Eq}_{i}}$ по следующей формуле:

$$
(x, \bar{x}) \in \widetilde{\operatorname{In}_{i}} \wedge x \equiv y\left(\widetilde{E_{i}}\right) \wedge \bar{x} \equiv \bar{y}\left(\widetilde{\mathrm{Eq}_{i}}\right) \quad \Longrightarrow \quad(y, \bar{y}) \in \widetilde{\operatorname{In}_{i}} ;
$$

5) автомат $\mathbf{A}=\operatorname{Atm}\left(\Pi_{1}, \Pi_{2}\right)$ изоморфен фактор-системе $\widetilde{\mathbf{A}} / \tilde{\theta}$ пятисортной алгебраической $\Omega$-системы

$$
\widetilde{\mathbf{A}}=\left(\left(X_{1}^{\prime}, \widetilde{D_{1}}, \widetilde{\operatorname{In}_{1}}\right),(S, \cdot),\left(X_{2}^{\prime}, \widetilde{D_{2}}, \widetilde{\operatorname{In}_{2}}\right), f_{1}, f_{2}\right)
$$

по конгруэнции $\tilde{\theta}=\left(\widetilde{E_{1}}, \widetilde{\mathrm{Eq}_{1}}, \Delta_{S}, \widetilde{E_{2}}, \widetilde{\mathrm{Eq}_{2}}\right)$, где $X_{1}^{\prime}=X_{2}^{\prime}=\widetilde{R Z}, \cdot$ - операция умножения полугруппы $S$ и операции $f_{i}: X_{1}^{\prime} \times S \rightarrow X_{i}^{\prime}$ определяются по формулам $f_{i}(x, s)=x \cdot s \in X_{i}^{\prime}(i=1,2)$;

6) для любого предложения $\Psi$ языка УИП $\mathbf{L}_{\mathbf{A}}$ эффективно строится такое предложение $\bar{\Psi}$ языка УИП $\mathbf{L}_{\mathbf{S}}$, что $\Psi$ в том и только том случае истинно на универсальном планарном автомате $\mathbf{A}$, если $\bar{\Psi}$ истинно на его полугруппе входных сигналов $\operatorname{Inp}(\mathbf{A})$.

ДокАЗАтеЛЬСтво. Рассмотрим универсальный планарный автомат $\mathbf{A}=$ $\operatorname{Atm}\left(\Pi_{1}, \Pi_{2}\right)$ над плоскостями $\Pi_{1}=\left(X_{1}, L_{1}\right), \Pi_{2}=\left(X_{2}, L_{2}\right)$ с полугруппой входных сигналов $S=\operatorname{Inp}(\mathbf{A})$.

Из теорем 1, 2 следует, что для такого автомата $\mathbf{A}$ выполняются равенства

$$
\begin{gathered}
\widetilde{R Z}=C, \quad \widetilde{E_{i}}=\varepsilon_{i}, \quad \overline{X_{i}}=\widetilde{R Z} / \widetilde{E_{i}}, \quad \widetilde{D_{i}}=C^{2} \backslash \varepsilon_{i}, \\
\widetilde{\mathrm{Eq}_{i}}=\eta_{i}, \quad \overline{L_{i}}=\widetilde{D_{i}} / \widetilde{\mathrm{Eq}_{i}}, \quad \overline{\rho_{i}}=\widetilde{\mathrm{In}_{i}} / \tilde{\theta},
\end{gathered}
$$

где $\widetilde{\operatorname{In}_{i}} / \tilde{\theta}=\left\{\left(\widetilde{E_{i}}(x), \widetilde{E q_{i}}(\bar{x})\right) \in \overline{X_{i}} \times \overline{L_{i}}:(x, \bar{x}) \in \widetilde{\operatorname{In}_{i}}\right\}$ и $i=1,2$. 
Следовательно, выполняются утверждения 1-4 и для определенного в теореме 1 автомата $\overline{\mathbf{A}}$ справедливы следующие равенства:

$$
\begin{aligned}
& \overline{\mathbf{A}}=\left(\bar{\Pi}_{1},(S, \cdot), \bar{\Pi}_{2}, \bar{\delta}, \bar{\lambda}\right)=\left(\left(\bar{X}_{1}, \bar{L}_{1}, \bar{\rho}_{1}\right),(S, \cdot),\left(\bar{X}_{2}, \bar{L}_{2}, \bar{\rho}_{2}\right), \bar{\delta}, \bar{\lambda}\right) \\
& =\left(\left(C / \varepsilon_{1}, D_{1} / \eta_{1}, \widetilde{\operatorname{In}_{1}} / \tilde{\theta}\right),(S, \cdot),\left(C / \varepsilon_{2}, D_{2} / \eta_{2}, \widetilde{\operatorname{In}_{2}} / \tilde{\theta}\right), \bar{\delta}, \bar{\lambda}\right) \\
& =\left(\left(\widetilde{R Z} / \widetilde{E_{1}}, \widetilde{D_{1}} / \widetilde{E q_{1}}, \widetilde{\operatorname{In}_{1}} / \tilde{\theta}\right),(S, \cdot),\left(\widetilde{R Z} / \widetilde{E_{2}}, \widetilde{D_{2}} / \widetilde{E q_{2}}, \widetilde{\operatorname{In}_{2}} / \tilde{\theta}\right), f_{1} / \tilde{\theta}, f_{2} / \tilde{\theta}\right)=\widetilde{\mathbf{A}} / \tilde{\theta},
\end{aligned}
$$

где $f_{1} / \tilde{\theta}\left(\widetilde{E_{1}}(x), s\right)=\widetilde{E_{1}}(x \cdot s), f_{2} / \tilde{\theta}\left(\widetilde{E_{1}}(x), s\right)=\widetilde{E_{2}}(x \cdot s)$ для элементов $x \in C$, $s \in S$. В силу теоремы 1 получаем, что $\mathbf{A} \cong \widetilde{\mathbf{A}} / \tilde{\theta}$ и выполняется утверждение 5 .

Доказательство утверждения 6 основывается на следующей эффективной синтаксической трансформации формул языка УИП $\mathbf{L}_{\mathbf{A}}$.

Для любого терма $t$ языка $\mathbf{L}_{\mathbf{A}}$ эффективно строится выражение $\bar{t}$ по следующему правилу:

- если $t=x^{(i)}-$ при $i=1$ индивидуальная переменная первого сорта (обозначающая точку плоскости состояний автомата), при $i=3$ индивидуальная переменная третьего сорта (обозначающая входной сигнал автомата) или при $i=4$ индивидуальная переменная четвертого сорта (обозначающая точку плоскости выходных сигналов автомата), то $\bar{t}=x$ - индивидуальная переменная языка $\mathbf{L}_{\mathbf{S}}$;

- если $t=x^{(i)}-$ при $i=2$ индивидуальная переменная второго сорта (обозначающая прямую плоскости состояний автомата) или при $i=5$ индивидуальная переменная пятого сорта (обозначающая прямую плоскости выходных сигналов автомата), то $\bar{t}=(x, y)$ - упорядоченная пара индивидуальных переменных языка $\mathbf{L}_{\mathbf{S}}$;

- если $t=t_{1}^{(3)} \cdot t_{2}^{(3)}$ для термов $t_{1}^{(3)}, t_{2}^{(3)}$ третьего сорта (обозначающих входные сигналы автомата), то $\bar{t}-$ терм $\overline{t_{1}^{(3)}} \cdot \overline{t_{2}^{(3)}}$ языка $\mathbf{L}_{\mathbf{S}}$;

- если $t=\delta\left(t^{(1)}, t^{(3)}\right)$ для терма $t^{(1)}$ первого сорта (обозначающего точку плоскости состояний автомата) и терма $t^{(3)}$ третьего сорта (обозначающего входной сигнал автомата), то $\bar{t}-$ терм $\overline{t^{(1)}} \cdot \overline{t^{(3)}}$ языка $\mathbf{L}_{\mathbf{S}}$;

- если $t=\lambda\left(t^{(1)}, t^{(3)}\right)$ для терма $t^{(1)}$ первого сорта (обозначающего точку плоскости состояний автомата) и терма $t^{(3)}$ третьего сорта (обозначающего входной сигнал автомата), то $\bar{t}-$ терм $\overline{t^{(1)}} \cdot \overline{t^{(3)}}$ языка $\mathbf{L}_{\mathbf{S}}$.

Пусть $\Psi-$ формула языка $\mathbf{L}_{\mathbf{A}}$ с бинарными предикатными символами $\epsilon_{1}$, $\epsilon_{2}$ и тремя бинарными функциональными символами $\cdot, \delta, \lambda$. Для такой формулы $\Psi$ эффективно построим формулу $\bar{\Psi}$ языка $\mathbf{L}_{\mathbf{S}}$ по следующему правилу:

- если $\Psi$ - атомарная формула $t_{1}=t_{2}$ для двух термов $t_{1}, t_{2}$ первого сорта (соответственно четвертого сорта), то $\bar{\Psi}$ - формула $\mathrm{E}_{1}\left(\overline{t_{1}}, \overline{t_{2}}\right)$ (соответственно $\left.\mathrm{E}_{2}\left(\overline{t_{1}}, \overline{t_{2}}\right)\right)$

- если $\Psi$ - атомарная формула $t_{1}=t_{2}$ для двух термов $t_{1}, t_{2}$ второго сорта (соответственно пятого сорта), то $\bar{\Psi}$ - формула $\mathrm{Eq}_{1}\left(\overline{t_{1}}, \overline{t_{2}}\right)$ (соответственно $\left.\mathrm{Eq}_{2}\left(\overline{t_{1}}, \overline{t_{2}}\right)\right)$

- если $\Psi$ - атомарная формула $t_{1}=t_{2}$ для двух термов $t_{1}, t_{2}$ третьего сорта, то $\bar{\Psi}-$ формула $\overline{t_{1}}=\overline{t_{1}}$;

- если $\Psi$ - атомарная формула $t_{1} \epsilon_{1} t_{2}$ (соответственно $t_{1} \epsilon_{2} t_{2}$ ) для терма $t_{1}$ первого сорта и терма $t_{2}$ второго сорта (соответственно для терма $t_{1}$ четвертого сорта и терма $t_{2}$ пятого сорта), то $\bar{\Psi}$ - формула $\operatorname{In}_{1}\left(\overline{t_{1}}, \overline{t_{2}}\right)$ (соответственно $\left.\operatorname{In}_{2}\left(\overline{t_{1}}, \overline{t_{2}}\right)\right)$;

- если $\Psi$ - формула $\Psi_{1} \star \Psi_{2}$ (где $\star-$ один из символов $\wedge, \vee, \Longrightarrow, \Longleftrightarrow$ ), то $\bar{\Psi}$ - формула $\bar{\Psi}_{1} \star \bar{\Psi}_{2}$; 
- если $\Psi-$ формула $\neg \Phi$, то $\bar{\Psi}-$ формула $\neg \bar{\Phi}$;

- если $\Psi$ - формула

$$
(\exists x) \Phi(x) \text { (соответственно }(\forall x) \Phi(x))
$$

для индивидуальной переменной $x$ первого или четвертого сорта, то $\bar{\Psi}-$ следующая формула языка $\mathbf{L}_{\mathbf{S}}$ :

$$
(\exists x)(R Z(x) \wedge \bar{\Phi}(x))(\text { соответственно }(\forall x)(R Z(x) \Longrightarrow \bar{\Phi}(x))) ;
$$

- если $\Psi$ - формула

$$
(\exists x) \Phi(x) \text { (соответственно }(\forall x) \Phi(x))
$$

для индивидуальной переменной $x$ второго или пятого сорта, то $\bar{\Psi}-$ следующая формула языка $\mathbf{L}_{\mathbf{S}}$ :

$$
\begin{gathered}
\left(\exists x_{1}, x_{2}\right)\left(R Z\left(x_{1}\right) \wedge R Z\left(x_{2}\right) \wedge \bar{\Phi}(\bar{x})\right) \\
\left(\text { соответственно }\left(\forall x_{1}, x_{2}\right)\left(R Z\left(x_{1}\right) \wedge R Z\left(x_{2}\right) \Longrightarrow \bar{\Phi}(\bar{x})\right)\right) ;
\end{gathered}
$$

- если $\Psi$ - формула

$$
(\exists x) \Phi(x) \text { (соответственно }(\forall x) \Phi(x))
$$

для индивидуальной переменной $x$ третьего сорта, то $\bar{\Psi}-$ следующая формула языка $\mathbf{L}_{\mathbf{S}}$ :

$$
(\exists x) \bar{\Phi}(x) \text { (соответственно }(\forall x) \bar{\Phi}(x)) \text {. }
$$

Для рассматриваемого автомата $\mathbf{A}=\operatorname{Atm}\left(\Pi_{1}, \Pi_{2}\right)$ и его полугруппы входных сигналов $S=\operatorname{Inp}(\mathbf{A})$ индукцией по числу связок и кванторов в формуле $\Psi$ языка $\mathbf{L}_{\mathbf{A}}$ легко обосновывается следующее свойство:

$$
\widetilde{\mathbf{A}} / \tilde{\theta} \models \Psi \quad \Longleftrightarrow \quad S \models \bar{\Psi} .
$$

С другой стороны, изоморфные алгебраические системы $\mathbf{A}$ и $\widetilde{\mathbf{A}} / \tilde{\theta}$ элементарно эквивалентны, т. е. для любой формулы $\Psi$ языка $\mathbf{L}_{\mathbf{A}}$ выполняется условие

$$
\mathbf{A} \models \Psi \quad \Longleftrightarrow \widetilde{\mathbf{A}} / \tilde{\theta} \models \Psi .
$$

В результате для любой формулы $\Psi$ языка $\mathbf{L}_{\mathbf{A}}$ получаем следующее условие:

$$
\mathbf{A} \models \Psi \quad \Longleftrightarrow \quad \operatorname{Inp}(\mathbf{A}) \models \bar{\Psi} .
$$

Отсюда следует, что выполняется утверждение 6.

Множество всех предложений языка $\mathbf{L}_{\mathbf{A}}$, истинных на планарном автомате $\mathbf{A}$, обозначается символом $\operatorname{Th}(\mathbf{A})$ и называется элементарной теорией автомата $\mathbf{A}$. Планарные автоматы $\mathbf{A}, \mathbf{A}^{\prime}$ называются элементарно эквивалентными, если их элементарные теории $\operatorname{Th}(\mathbf{A}), \operatorname{Th}\left(\mathbf{A}^{\prime}\right)$ совпадают, т. е. $\operatorname{Th}(\mathbf{A})=$ $\operatorname{Th}\left(\mathbf{A}^{\prime}\right)$. Это означает, что такие автоматы $\mathbf{A}, \mathbf{A}^{\prime}$ не могут быть различимы с помощью свойств, выраженных формулами языка УИП $\mathbf{L}_{\mathbf{A}}$.

Ясно, что из элементарной эквивалентности планарных автоматов $\mathbf{A}, \mathbf{A}^{\prime}$ следует элементарная эквивалентность полугрупп входных сигналов этих автоматов. Обратное утверждение для универсальных планарных автоматов обосновывается с помощью приведенной выше элементарной определимости класса универсальных планарных автоматов в классе всех полугрупп. 
Теорема 4. Для плоскостей $\Pi_{1}, \Pi_{2}, \Pi_{1}^{\prime}, \Pi_{2}^{\prime}$ универсальные планарные автоматы $\operatorname{Atm}\left(\Pi_{1}, \Pi_{2}\right)$ и $\operatorname{Atm}\left(\Pi_{1}^{\prime}, \Pi_{2}^{\prime}\right)$ элементарно эквивалентны в том и только том случае, если элементарно эквивалентны полугруппы входных сигналов этих автоматов.

ДокАЗАТЕЛЬСтво. По теореме 3 существуют формулы языка УИП $\mathbf{L}_{\mathbf{S}}, \mathrm{c}$ помощью которых для универсального планарного автомата $\mathbf{A}=\operatorname{Atm}\left(\Pi_{1}, \Pi_{2}\right)$ на его полугруппе входных сигналов $S=\operatorname{Inp}(\mathbf{A})$ строится изоморфная автомату $\mathbf{A}$ алгебраическая фактор-система $\widetilde{\mathbf{A}} / \tilde{\theta}$. При этом для любого предложения $\Psi$ языка УИП $\mathbf{L}_{\mathbf{A}}$ эффективно строится такое предложение $\bar{\Psi}$ языка УИП $\mathbf{L}_{\mathbf{S}}$, что $\Psi$ в том и только том случае истинно на универсальном планарном автомате $\mathbf{A}$, если $\bar{\Psi}$ истинно на его полугруппе входных сигналов $S$, т. е. выполняется условие

$$
\mathbf{A} \models \Psi \quad \Longleftrightarrow \tilde{\mathbf{A}} / \tilde{\theta} \models \Psi \quad \Longleftrightarrow S \models \bar{\Psi}
$$

С другой стороны, с помощью упомянутых выше формул для универсального планарного автомата $\mathbf{A}^{\prime}=\operatorname{Atm}\left(\Pi_{1}^{\prime}, \Pi_{2}^{\prime}\right)$ на его полугруппе входных сигналов $S^{\prime}=\operatorname{Inp}\left(\mathbf{A}^{\prime}\right)$ строится изоморфная автомату $\mathbf{A}^{\prime}$ алгебраическая факторсистема $\widetilde{\mathbf{A}^{\prime}} / \tilde{\theta}^{\prime}$. При этом для любого предложения $\Psi$ языка УИП $\mathbf{L}_{\mathbf{A}}$ эффективно строится такое предложение $\bar{\Psi}$ языка УИП $\mathbf{L}_{\mathbf{S}}$, что $\Psi$ в том и только том случае истинно на универсальном планарном автомате $\mathbf{A}^{\prime}$, если $\bar{\Psi}$ истинно на его полугруппе входных сигналов $S^{\prime}$, т. е. выполняется условие

$$
\mathbf{A}^{\prime} \models \Psi \quad \Longleftrightarrow \widetilde{\mathbf{A}^{\prime}} / \tilde{\theta^{\prime}} \models \Psi \quad \Longleftrightarrow \quad S^{\prime} \models \bar{\Psi}
$$

Так как из элементарной эквивалентности полугрупп входных сигналов $S, S^{\prime}$ следует

$$
S \models \bar{\Psi} \quad \Longleftrightarrow \quad S^{\prime} \models \bar{\Psi},
$$

выполняется

$$
\mathbf{A} \models \Psi \quad \Longleftrightarrow \quad \mathbf{A}^{\prime} \models \Psi
$$

т. е. автоматы $\mathbf{A}, \mathbf{A}^{\prime}$ элементарно эквивалентны.

Пусть Inp - класс всех полугрупп входных сигналов универсальных планарных автоматов. Для класса планарных автоматов $\mathbf{K}$ символом $\operatorname{Inp}(\mathbf{K})$ обозначается класс полугрупп вида $\operatorname{Inp}(\mathbf{A})$, где $\mathbf{A} \in \mathbf{K}$. Класс планарных автоматов $\mathbf{K}$ называется (конечно) Inp-аксиоматизируемым, если класс полугрупп $\operatorname{Inp}(\mathbf{K})$ относительно (конечно) аксиоматизируем в классе Inp, т. е. выполняется равенство $\operatorname{Inp}(\mathbf{K})=\mathbf{I n p} \cap \mathbf{S}^{\prime}$ для некоторого (конечно) аксиоматизируемого класса полугрупп $\mathbf{S}^{\prime}$.

С помощью приведенной выше элементарной определимости класса универсальных планарных автоматов в классе всех полугрупп получаем следующий результат.

Теорема 5. Любой (конечно) аксиоматизируемый класс универсальных планарных автоматов (конечно) Inp-аксиоматизируем.

ДокАзАтЕльство. В теореме 3 определено такое преобразование $\Psi \mapsto \bar{\Psi}$ формул $\Psi$ языка УИП $\mathbf{L}_{\mathbf{A}}$ в формулы $\bar{\Psi}$ языка УИП $\mathbf{L}_{\mathbf{S}}$, что для любого универсального планарного автомата $\mathbf{A}=\operatorname{Atm}\left(\Pi_{1}, \Pi_{2}\right)$ с полугруппой входных сигналов $S=\operatorname{Inp}(\mathbf{A})$ выполняется условие

$$
\mathbf{A} \models \Psi \quad \Longleftrightarrow \quad \operatorname{Inp}(\mathbf{A}) \models \bar{\Psi} .
$$


Следовательно, если некоторый класс универсальных планарных автоматов $\mathbf{K}$ аксиоматизируется множеством формул $\Sigma$, то в силу теоремы 3 класс его полугрупп входных сигналов $\operatorname{Inp}(\mathbf{K})$ относительно аксиоматизируется в классе Inp множеством формул $\bar{\Sigma}=\{\bar{\Psi}: \Psi \in \Sigma\}$. Ясно, что для конечного множества аксиом $\Sigma$ класса автоматов $\mathbf{K}$ множество формул $\bar{\Sigma}$ также будет конечным.

Например, с помощью трансформации соответствующих аксиом проективных и аффинных плоскостей легко показать, что классы универсальных планарных автоматов над проективными плоскостями и над аффинными плоскостями конечно Inp-аксиоматизируемы.

\section{5. Заключение}

Из [21] следует, что главным инструментом решения проблемы представимости универсальных планарных автоматов входными сигналами являются канонические отношения автоматов. В настоящей работе показано, что эти отношения определяются формулами логики первого порядка в полугруппах входных сигналов таких автоматов и доказана элементарная определимость класса универсальных планарных автоматов в классе полугрупп. Полученные результаты позволяют исследовать взаимосвязь между элементарными свойствами универсальных планарных автоматов и их полугрупп входных сигналов, проанализировать взаимосвязь важных свойств элементарных теорий классов планарных автоматов и элементарных теорий классов полугрупп таких, как проблема элементарной определимости универсальных планарных автоматов их полугруппами входных сигналов, проблема алгоритмической разрешимости элементарных теорий классов универсальных планарных автоматов и др.

Благодарность. Автор признателен рецензенту этой статьи за его полезные замечания.

\section{ЛИТЕРАТУРА}

1. Плоткин Б. И. Группы автоморфизмов алгебраических систем. М.: Наука, 1966.

2. Пинус А. Г. Об элементарной эквивалентности производных структур свободных решеток // Изв. вузов. Математика. 2002. № 5. С. 44-47.

3. Пинус А. Г. Об элементарной эквивалентности производных структур свободных полугрупп, унаров и групп // Алгебра и логика. 2004. Т. 43, № 6. С. 730-748.

4. Пинус А. Г. Об элементарной эквивалентности решеток подалгебр и групп автоморфизмов свободных алгебр // Сиб. мат. журн. 2008. Т. 49, № 4. С. 865-869.

5. Важенин Ю. М. Элементарные свойства полугрупп преобразований упорядоченных множеств // Алгебра и логика. 1970. Т. 9, № 3. С. 281-301.

6. Важенин Ю. М. Об элементарной определяемости и элементарной характеризуемости классов рефлексивных графов // Изв. вузов. Математика. 1972. № 7. С. 3-11.

7. Глускин Л. М. Полугруппы изотонных преобразований // Успехи мат. наук. 1961. Т. 16, № 5. С. $157-162$.

8. Глускин Л. М. Полугруппы и кольца эндоморфизмов линейных пространств // Изв. АН СССР. Сер. мат. 1959. Т. 23. С. 841-870.

9. Марков В. Т., Михалев А. В., Скорняков Л. А., Туганбаев А. А. Кольца эндоморфизмов модулей и структуры подмодулей // Алгебра. Топология. Геометрия. М.: ВИНИТИ, 1983. С. 183-254. (Итоги науки и техники; Т. 21).

10. Важенин Ю. М., Пинус А. Г. Элементарная классификация и разрешимость теорий производных структур // Успехи мат. наук. 2005. Т. 60, № 3. С. 3-40.

11. Бунина Е. И., Михалев А. В. Элементарная эквивалентность колец эндоморфизмов абелевых $p$-групп // Фунд. и прикл. математика. 2004. Т. 10, № 2. С. 135-224.

12. Мурзин Ф. А. Об элементарной эквивалентности колец непрерывных функций // Теория моделей и ее приложения. Алма-Ата: Казахский гос. университет, 1980. С. 72-74. 
13. Ершов Ю. Л. Проблемы разрешимости и конструктивные модели. М.: Наука, 1980.

14. Плоткин Б. И., Гринглаз Л. Я., Гварамия А. А. Элементы алгебраической теории автоматов. М.: Высшая школа, 1994.

15. Molchanov V. A. Semigroups of mappings on graphs// Semigroup Forum. 1983. V. 27. P. $155-199$.

16. Свердловская тетрадь: Сборник нерешенных проблем теории полугрупп. Свердловск: Урал. гос. ун-т, 1979.

17. Molchanov V. A. A universal planar automaton is determined by its semigroup of input symbols // Semigroup Forum. 2011. V. 82. P. 1-9.

18. Улам С. Нерешенные математические задачи. М.: Наука, 1964.

19. Ершов Ю. Л., Палютин Е. А. Математическая логика. М.: Наука, 1987.

20. Хартсхорн Р. Основы проективной геометрии. М.: Мир, 1970.

21. Молчанов B. А. Представление универсальных планарных автоматов входными сигналами // Изв. Сарат. ун-та. Нов. сер. Математика. Механика. Информатика. 2013. Т. 13, № 2 : 2. C. $31-37$.

22. Молчанов В. А. Абстрактная характеризация полугрупп входных сигналов универсальных планарных автоматов // Изв. Сарат. ун-та. Нов. сер. Математика. Механика. Информатика. 2015. Т. 15, № 1. С. 113-121.

Поступила в редакиию 5 марта 2019 2.

После доработки 8 апреля 2019 г.

Принята к публикаиии 15 мая 2019 г.

Молчанов Владимир Александрович

Саратовский государственный университет им. Н. Г. Чернышевского, ул. Астраханская, 83, Саратов 410012

v.molchanov@inbox.ru 